\title{
Treatment and prevention of childhood obesity
}

Ceylon Medical Journal 2011; 56: 77-80

Obesity is defined as accumulation of excess fat in body associated with adverse health outcomes [1]. Although consensus is lacking, a body fat content of more than $20-25 \%$ in boys and $25-32 \%$ in girls are associated with morbidity [2,3]. Socioeconomic advancements leading to nutritional transition from a traditional diet to a high fat, refined carbohydrate containing diet accompanied by sedentary behaviour has made childhood obesity an emerging problem in many developing countries.

Effects of obesity are both physical and psychological. It could affect any organ of the body either metabolically (diabetes mellitus, cardiovascular disease, NAFLD/NASH, metabolic syndrome, glomerular disease, polycystic ovarian syndrome, gall stones and pseudotumour cerebri) or mechanically (slipped capital femoral epiphysis, genu alga, tibia vara, flat foot, scoliosis and osteoarthritis). Psychological effects are bullying, peer rejection, lack of friends, lack of self confidence and low job opportunities [4]. Obesity occurs due to surplus of energy being deposited as fat in the body. The only way of preventing excess calorie deposition is by utilising them through physical activity.
In clinical practice body mass index (BMI) is used as a convenient surrogate marker to diagnose obesity. However, current BMI cut-off values are not sensitive in detecting obesity in Sri Lankan children mainly due to the high fat content South Asians have for any given BMI compared to many other ethnic groups [5]. BMI "tracks" from about 6 years of age into adult life. A small increase of BMI in childhood will lead to a larger difference during adult life and it is resistant to change [6]. The danger of childhood obesity is that it persists into adulthood and related pathological changes begin from childhood leading to non communicable diseases and complications at a younger age. Therefore early detection and correction of childhood obesity is of paramount importance.

\section{Diagnosis}

Obesity is diagnosed when BMI is $>95$ th centile (or $>2 \mathrm{SD})$. BMI between 85th to 95th centile ( +1 to $+2 \mathrm{SD})$ is considered as overweight or at risk of obesity. Gender specific BMI for age and WHO growth charts can be used for calculation of BMI (http://www.who.int/childgrowth/ en/). Both groups need complete clinical evaluation and management (Figure 1).

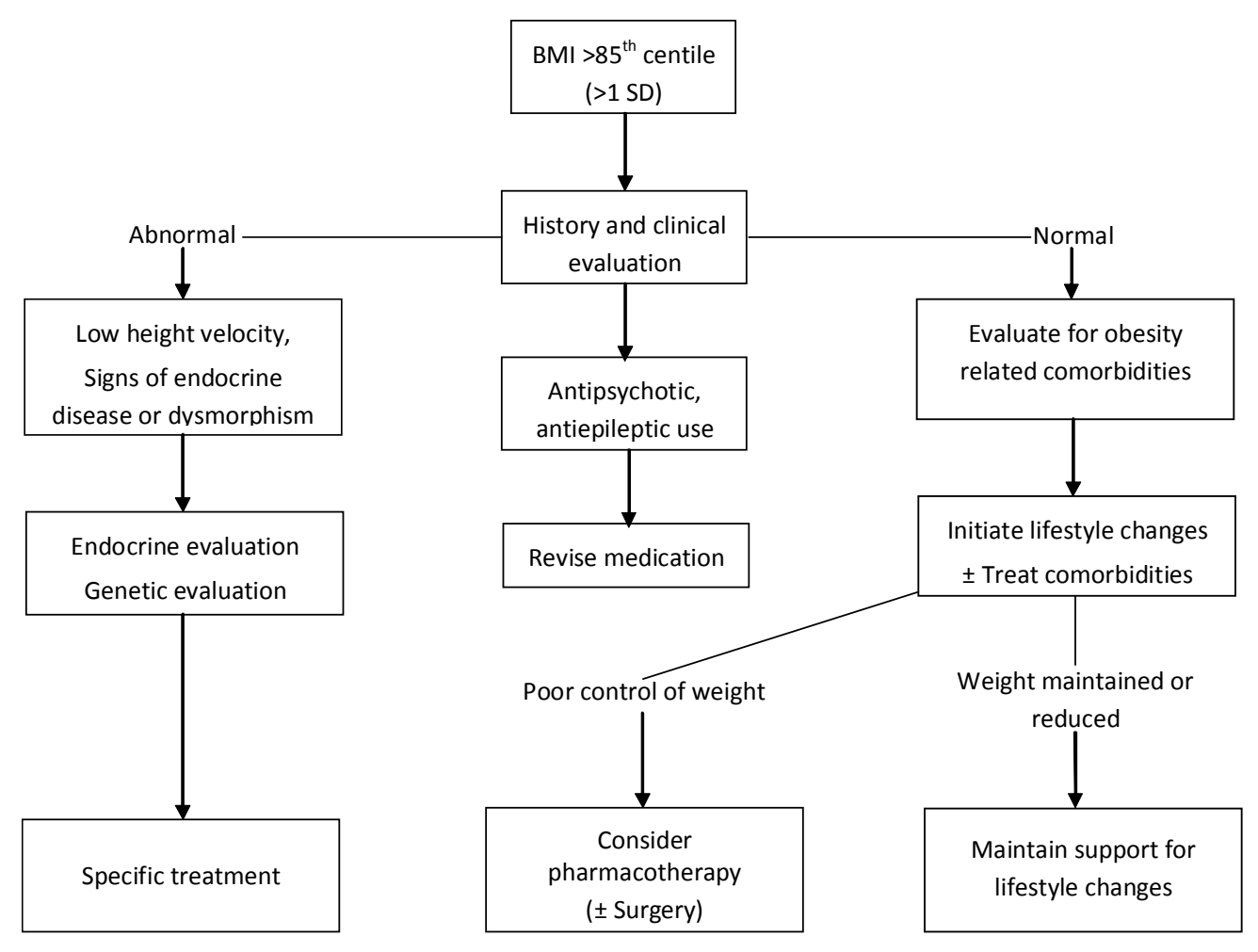

Figure 1. Diagnosis and management flow chart. 


\section{Aetiology}

Majority of cases is due to excess calorie intake and is known as simple obesity. Only a minority are due to pathological causes. They could be due to a syndrome (Bardet Biedel, Alstrom, Prader Willi, Down) or an endocrine disorder (hypothyroidism, growth hormone deficiency, Cushing disease/syndrome, pseudohypoparathyroidism). In simple obesity, both weight and height increase and the distribution in weight for age and height for age charts are similar similar (both above median). However, as a rule of thumb, pathological obesity results in a short obese child (height below median). Exceptions are adrenal tumour with concomitant hypersecretion of androgens and cortisol and the condition termed growth without growth hormone which is a rare condition seen in central nervous system pathologies [4]. Clinical history and examination will help in identifying these conditions. Leptin deficiency, although a pathological condition, behaves like simple obesity as the effects are brought by excess calorie intake. When it is clinically suggestive of simple obesity, investigations for pathological aetiology (genetic and endocrinological evaluation) can be deferred.

\section{Clinical evaluation}

A complete clinical history including birth history (weight, maturity, maternal illnesses), family history (obesity in parents and siblings, diabetes and cardiovascular disease), medical history (sleep apnoea, polydypsia, polyuria, recent weight loss, acne, hirsuitism and menstrual history in a pubertal girl suggestive of polycystic ovary syndrome, school performance, day time somnolence) are important to identify aetiology and complications. Although unreliable and subject to error, detailed feeding history is important to outline the management. Information about type, quantity and frequency of beverages, snacks, fast food and outside meals taken are important in the management. Detailed activity history should include frequency and duration spent on physical activity both at home and school, amount of structured activities including participating in team sports and forms of physical activity engaged in day to day life. Another important aspect of activity history is information on sedentary behaviour (spent in front of television, video games and computers). It is a way of spending time with minimum consumption of energy. Drug history is important as antipsychotic medication (risperidone) and antiepileptics (sodium valproate) are associated with obesity.

Complete physical evaluation including waist circumference measurement (measured in the horizontal plane at the level of midpoint between iliac crest and costal margin in the mid-axillary line), blood pressure measurement using correct size cuff and interpreting using appropriate tables, examining for acanthosis and clinical features suggestive of endocrine or syndromic causes is useful in determining the aetiology and complications $[7,8]$.

\section{Investigations}

In an obese child (above 5 years) with the clinical suspicion of simple obesity, basic investigations are performed to evaluate associated comorbidities rather than to identify the aetiology. Fasting blood sugar, lipid profile and amino transferase levels (ALT and AST) are done after a 12-hour overnight fast. A load of anhydrous glucose $1.75 \mathrm{~g} / \mathrm{kg}$ body weight to a maximum of $75 \mathrm{~g}$ is given dissolved in water (taken within 5 minutes) and blood will be drawn for random blood sugar at the end of 2 hours (Table 1). Investigations in an obese child below 5 years of age are usually done only when there is severe obesity with strong family history of comorbidities or resistant to initial therapy.

Table 1. Cutoff values for diagnosing impaired glucose homeostasis and dyslipidaemia

\begin{tabular}{ll}
\hline Comorbidity & Cutoff value \\
\hline Impaired fasting glucose & FBS $>100 \mathrm{mg} / \mathrm{dl}$ but $<126 \mathrm{mg} / \mathrm{dl}$ \\
Impaired glucose tolerance & 2-h OGTT $>140 \mathrm{but}<200 \mathrm{~g} / \mathrm{dl}$ \\
Diabetes mellitus & FBS $\geq 126 \mathrm{mg} / \mathrm{dl}$ or 2 -h OGTT $\geq 200 \mathrm{mg} / \mathrm{dl}$ \\
Dyslipidaemia & \\
$\quad$ Triglyceride & $\geq 160 \mathrm{mg} / \mathrm{dl}$ 90th centile \\
& $\geq 150 \mathrm{mg} / \mathrm{dl}$ (cutoff for metabolic syndrome[13]) \\
& $\geq 200 \mathrm{mg} / \mathrm{dl}$ (90th centile) \\
Total cholesterol & $\geq 130 \mathrm{mg} / \mathrm{dl}$ (90th centile) \\
LDL cholesterol & $\leq 35 \mathrm{mg} / \mathrm{dl}$ (25th centile) \\
HDL cholesterol &
\end{tabular}

Conversion of $\mathrm{mg} / \mathrm{dl}$ to $\mathrm{mmol} / \mathrm{l}$; glucose $\times 0.5555$, cholesterol $\times 0.0259$, triglyceride $\times 0.0113$ 
Ultrasound scan of abdomen will help in detecting hepatic steatosis or non alcoholic fatty liver disease (NAFLD) and in the presence of an elevated ALT and high ALT: AST ratio it is suggestive of non alcoholic steato hepatitis (NASH), provided there had been no other illness accountable for hepatitis within the recent past. Persistence of elevated ALT for more than 6 months requires further investigations for an underlying liver pathology and warrants a liver biopsy for a definitive diagnosis. Features suggestive of a genetic or endocrine disorder warrants special investigations and /or referral to a specialised centre. Bone age is advanced in simple obesity but matches the height age of the child. Investigations need to be repeated once in every 6-12 months depending on the degree of abnormality and response to management.

\section{Management}

The mainstay of management is behaviour modification and it should involve the whole family. Targets need to be set and would depend on the pubertal stage. Ultimate goal of management is to achieve an appropriate weight for height (BMI) and control/prevent any complications. The initial target for children who have not undergone the pubertal growth spurt (Tanner stage $\leq 2$ in girls and $3 \leq$ in boys) is to maintain weight till the pubertal growth spurt occurs (minimum of 2 years). When the pubertal growth spurt occurs while maintaining the weight, the BMI will decrease. However, if weight could be reduced it is a bonus. The BMI should reach below 85th centile (1SD). Children who have passed the pubertal growth spurt need to lose weight to achieve a satisfactory BMI. Although most of the targets are achieved during the initial phase of management, sustaining them over a long period of time is not easy. Therefore a gradual change in behaviour over a long period of time is more practical and should involve changes in the whole family.

As children are growing it is important to provide adequate calories to prevent protein catabolism. Micronutrient deficiencies as well as essential fatty acid deficiencies should be avoided. Providing about $80 \%$ of the normal calorie requirement is appropriate. Selecting high fiber diet with low glycaemic index food is important. Fruit and vegetable intake, minimum of 5 portions a day, should be increased. Intake of refined carbohydrates and fatty foods should be restricted. Non fat milk is recommended above 2 years of age as children under 2 years are more prone to develop essential fatty acid deficiencies. A dietician's advice would be useful.

Rather than banning food items, the policy should be to allow them in a restricted manner. Food based portion sizes can be used as a guide to determine the amount of food that each child should receive $[9,10]$. The practical way of avoiding consumption of high calorie containing refined food is by avoiding stocking them at home. Therefore the cooperation of the entire family is needed and by doing so it will help preventing obesity/overweight among other members as well. Taking the meals at a regular time with whole family together is important. Meal times should be short and "grazing" throughout the day should be avoided. Fizzy sweetened beverages should be replaced by water. This will not only help in reducing obesity, but also improve dental health.

\section{Physical activity}

Physical activity will not only help in reducing weight but also improve body composition and cardiovascular fitness. Minimum of 60 minutes of moderate to vigorous physical activity is recommended [4]. At the onset many children would be unable to engage in physical activity for such an extent of time due to fatigue and would give up. The thick subcutaneous fat layer prevents dissipation of heat leading to easy fatigue. Therefore in the initial stages they should engage in physical activity for short time spells alternating with relaxation. However, the exercise session should continue for the entire period (60 minutes) although the cumulated exercise time would be well under 60 minutes. Once the subcutaneous fat layer thins and endurance builds up children will be able to engage in physical activity for longer periods with less relaxation. Physical activity alone does not bring down the weight. Calorie restriction is important and they should not be given calorie rich beverages and snacks especially after exercise. Similarly children engaged in competitive sports should cut down calories during the off season.

As much as engaging in physical activity, limiting all sedentary activity (TV viewing, computer and play station usage) to a maximum of 1-2 hours per day is important. It is important for children to get involved in day to day household activities and restrict the use of automated appliances.

\section{Psychosocial issues}

Main difficulty in management of obese children is motivating them as well as parents to adhere to the management plan. Parents should be educated about healthy rearing practices related to diet and activity. It will help not only treating the patient but preventing obesity in other members of the family and improving the quality of life of entire family. Parents should set an example of behaviour (food and physical activity) for children to follow. It is important to teach children coping skills as they are constantly being bullied by peers.

\section{Pharmacological therapy}

Pharmacotherapy is indicated only when behaviour modification programmes have failed to achieve any control and severe comorbidities exist. Poor weight control with strong family history of type 2 diabetes mellitus and, cardiovascular risk strengthen the case to start medication. Although several agents are available only sibutramine, orlistat and metformin are commonly used [4]. 


\section{Bariatric Surgery}

Bariatric surgery is used in a highly selective manner during childhood. It will be considered only when a satisfactory behaviour modification programme with pharmacological agents has failed. Commonly agreed cutoffs are BMI $>40 \mathrm{~kg} / \mathrm{m}^{2}$ with severe comorbidities or $>50 \mathrm{~kg} / \mathrm{m}^{2}$ with resistance to therapy [4].

\section{Prevention of obesity}

Preventive measures should begin from early infancy. Data have shown that early rapid growth leads to development of obesity and comorbidities later in life [11]. Especially excessive feeding of term low birth weight infants to achieve a catch up growth is detrimental [11]. Therefore it is important to have strict growth monitoring and provide the required nutrients to let the child grow along the same trajectory that he has been growing during intra uterine life. Breast feeding has shown to have a protective effect on later obesity. Media should be used to educate parents and school based programmes should be used to educate children about healthy lifestyle behaviour. Clinicians need to pursue an active case detection policy and once a child is identified, steps should be taken to address the whole family as others could already be obese or are highly vulnerable to develop obesity.

Clinicians should play an advocacy role in drawing up regulatory policies to prevent targeting of children by unhealthy food promotion, improving quality of food at schools and implementing school canteen policy and providing the community with opportunities of safe walking, cycling and recreational facilities.

\section{References}

1. WHO Obesity: preventing and managing the global epidemic. WHO Technical Report Series 894, WHO Geneva. 2000.

2. Dwyer T, Blizzard CL. Defining obesity in children by biological endpoint rather than population distribution. International Journal of Obesity 1996; 20: 472-80.

\section{P Wickremasinghe ${ }^{1}$}

${ }^{1}$ Department of Paediatrics, Faculty of Medicine, University of Colombo, Sri Lanka.

Correspondence: VPW, e-mail <pujithaw@yahoo.com>.
3. Lohman TG. The prevalence of obesity in children in the United States. In: Advances in body composition assessment. Human Kinetics, Champaign, IL. Monogram 1992; 3: 79-89.

4. August GP, Caprio S, Fennoy I, et al. Prevention and treatment of paediatric obesity: an endocrine society clinical practice guideline based on expert opinion. Journal of Clinical Endocrinology and Metabolism 2008; 93: 4576-99.

5. Wickramasinghe VP, Lamabadusuriya SP, Cleghorn GJ, Davies PSW. BMI as a measure of obesity in Sri Lankan children: validity of currently used cut off values. Ceylon Medical Journal 2009; 54:114-9.

6. Guo SS, Roche AF, Chumlea WC, Gardner JD, Siervogel RM. The predictive value of childhood body mass index values for overweight at age 35 years. American Journal of Clinical Nutrition 1994; 59: 810-9.

7. McCarthy HD, Jarrett KV, Crawley HF. The development of waist circumference percentiles in British children aged 5.0-16.9 years. European Journal of Clinical Nutrition 2001; 55: 902-7.

8. National High Blood Pressure Education Programme Working Group on High Blood Pressure in Children and Adolescents. The fourth report on the diagnosis, evaluation, and treatment of high blood pressure in children and adolescents. Paediatrics 2004; 114: 555-76.

9. Ministry of Health, Nutrition and Welfare. Food Based Dietary Guidelines for Sri Lankans (2002) Colombo, Sri Lanka.

10. Gidding SS, Dennison BA, Birch LL, et al. Dietary recommendations for children and adolescents: a guide for practitioners. Paediatrics 2006; 117: 544-59.

11. Lucas, A. Growth and later health: a general perspective. In Lucas A, Makrides M, Ziegler EE eds. Importance of Growth for Health and Development. Nestle Nutrition Institute Workshop Series Paediatric Programme. Switzerland. Karger 2010; 65: 1-11.

12. Hickman TB, Briefel RR, Carroll MD, Rifkind BM, Cleeman JI. Distributions and trends of serum lipid levels among United States children and adolescents aged 4-19 years: data from the Third National Health and Nutrition Examination Survey. Preventive Medicine 1998; 27: 879-90.

13. Zimmet P, Alberti KGMM, Kaufman F, et al. The metabolic syndrome in children and adolescents - an IDF consensus report. Paediatric Diabetes 2007; 8: 299-306. 